\title{
Analyzing the effect of MicroRNAs on Hepatogenic differentiation of Mesenchymal stem cells
}

\author{
Ehsan Jahangirian*, Seyed Moayed Alavian
}

Baqiyatallah Research Center for Gastroenterology and Liver Disease Baqiyatallah University of Medical Sciences, Tehran, Iran

\section{A R T I C L E I N F O}

\section{Article history:}

Received 18 May 2016

Received in revised form

25 July 2016

Accepted 26 July 2016

\section{Keywords:}

Hepatogenic

Mesenchymal stem cells

MicroRNAs

\begin{abstract}
A B S T R A C T
This research aims at analyzing the effect of microRNAs on hepatogenic Differentiation of mesenchymal stem cells. According to the results, miR1246, miR-1290, miR-148a, miR-30a, miR-424 and miR-542-5p were definitely overexpressed during hepatic differentiation of hMSCs. Also the results indicate that ectopic overexpression of miR-122 cannot initiate hepatic differentiation of hMSCs. In other words, hMSCs can be converted into functional hepatocytes only if all the miRs (miR-122, miR-1246, miR1290, miR-148a, miR-30a, miR-424, and miR-542-5p) are used.
\end{abstract}

(C) 2016 The Authors. Published by IASE. This is an open access article under the CC BY-NC-ND license (http://creativecommons.org/licenses/by-nc-nd/4.0/).

\section{Introduction}

According to the main question of the recent research regarding the effect of MicroRNAs on the hepatogenic differentiation of mesenchymal stem cells, some miRNAs are encoded by unique genes (intergenic miRNAs) (Costa, 2010; Krol et al., 2010) and others are embedded into the intronic regions of protein-coding genes (intragenic miRNAs) (Sontheimer et al., 2005). Samples of intergenic miRNA are miR-210, miR-10a, miR-21, and miR$222 /$ miR-22. They are encoded by unique genes located in chromosomes 11, 17, 17, 6 and X, respectively. The transcription process of RNA polymerase II of the miR genes increases the initial miRNAs (pri-miRNAs) which have typical stem-loop structures (Callegari et al., 2014). They are rapidly processed by the nuclear RNase endonuclease-III Drosha which eliminates the branches and gives rise to precursor miRNAs (pre-miRNA) of around 60100 nts in length. A good example of intragenic miRNA is miR-301. Its genomic sequences are embedded into the intronic regions of ska2. In this unique case, the transcription of miRNA sequences relies on the function of the cellular promoter of the host gene. The miR sequences follow the splicing pathways giving rise to a "Mirtron" (microRNA/ intron) sequence further processed by debranching enzyme to generate a pre-miRNA. The microRNA transcription could be controlled by targeting

\footnotetext{
* Corresponding Author.

Email Address: ehsanjahangirian20@gmail.com (E. Jahangirian)

http://dx.doi.org/10.21833/ijaas.2016.07.011

2313-626X/C) 2016 The Authors. Published by IASE.

This is an open access article under the CC BY-NC-ND license

(http://creativecommons.org/licenses/by-nc-nd/4.0/)
}

regulatory transcription factors, the microRNA promoter itself, or the promoter of the host gene (Finch et al., 2014). According to Xi et al., (2010) the knocking-down process of C-EBP-b decreases the recruitment with this transcription factor on the promoter of the LOC554202 gene (hosting miR-31) and down-regulation of miR-31 in a remarkable manner. Another example is that the knockdown of the transcription factor Foxo3a increases miR-21 levels consistent with the function of this transcription factor which regulates miR-21 transcription in a negative manner. In both cases of intergenic miRNAs and intragenic miRNAs, the premiRNAs are transported from the nucleus to the cytoplasm by exportin-5. In the cytoplasm, premiRNAs are further processed by another RNase endonuclease-III (Dicer) to generate mature miRNAs. Probably the most trusted approach to alter processing or targeting functions of microRNA is the antisense strategy that have been reported in many papers. Considering this strategy, microRNAs have already been targeted by anti-miR molecules. This antisense strategy appears to be more specific according to the process of targeting transcription factors or miRNA promoters since it affects single microRNAs or microRNA families. MicroRNAs (miRNAs) are small non-coding RNA molecules (20$24 \mathrm{bp}$ in length) that regulate gene expression in a negative manner through binding to complementary sequences typically residing within the 39 untranslated regions (UTR) of mRNAs. Partial sequence complementarity between miRNA and the target mRNA results in repression of mRNA translation, whereas a higher amount of sequence complementarity can guide destruction of the target mRNA. Already more than 1000 human miRNA 
precursor sequences have already been deposited in miR Base and more than $50 \%$ of cellular mRNAs have already been estimated to be under miRNA regulation. miRNAs are expressed in tissue and differentiation state-specific patterns and in many cases are expressed or deleted in different types of human cancers (Kane et al., 2014). The basic mechanism leads to the alteration of gene expression. This technique occurs in the cytoplasm where in fact the pre-miRNA hairpin is cleaved by the RNase III enzyme Dicer which interacts with the 30 end of the hairpin and cuts away the loop joining the 30 and 50 arms, yielding an imperfect miRNA/ miRNA duplex. One of the main strands is incorporated into the RISC where it binds to focus on mRNA sequences (Fig. 1). Animal miRNAs are often complementary to a niche site in the 30UTR. Perfect or relatively perfect base pairing with the target RNA, promotes cleavage of the RNA. Note that the presence of nucleotides 2-7 of the miRNA (the seed region) is essential. These nucleotides help recognizing the functions of microRNAs. Here is the key process permitting mature miRNAs to exert their effects in gene regulation. The ultimate effect of miRNAs activity is considered as the inhibition of the synthesis of the protein(s) encoded by the target mRNA(s). Since a single 30UTR of a given mRNA contains signal sequences for a number of microRNAs, applied biological studies are required to ascertain which microRNA must certainly be targeted to attain alteration of gene expression. Therefore, it is necessary to analyze the possible effects on the expression of other mRNA targets. An alteration of a single microRNA may exhibit multiple effects especially in combination with the targeting activity of other miRNAs enabling the achievement of strong biological effect.

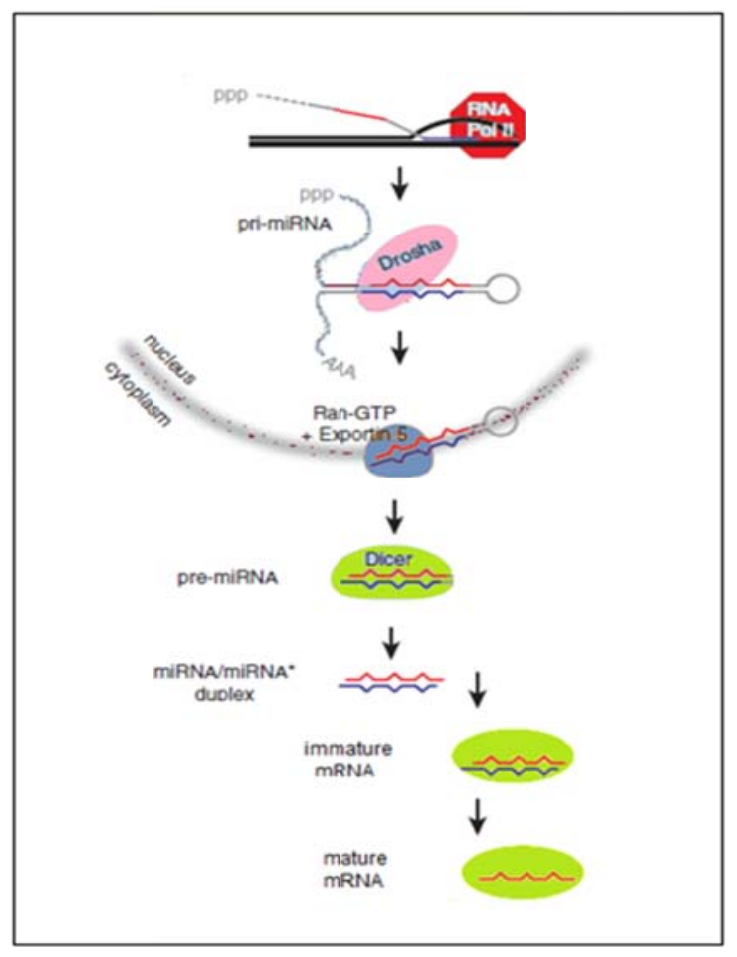

Fig. 1: miRNA biogenesis

\subsection{MicroRNAs in the liver}

The liver can be considered as one of the biggest organs in the human body. Its multiple functions can be mentioned as detoxification (e.g. urea and protein-bound toxins), synthesis (e.g. proteins and proteins such as for example as an example growth factors and albumin), and regulation (e.g. acid-basehomeostasis and the vitality way to obtain the central nervous system). Acute or acute-on-chronic failure of the liver forms a life-threatening situation. Liver transplantation often remains the only real opportunity to rescue the in-patient. Within the last few years an increasing body of evidence indicates that in addition to transcriptional gene regulation, posttranscriptional processes have pivotal roles in cellular gene regulatory ne:tworks. These molecules inhibit protein synthesis and only one miRNA has the capacity to target on around several hundred mRNAs. Studies reveal that specific signatures of expressed miRNAs are related to many physiological and pathological processes. For example, Tzur et al. (2009) identified specific miRNA expression profiles during different stages of human hepatic development (Wang et al., 2009). Other studies have focused on the role of miRNAs during hepatic carcinogenesis and hepatic regeneration following partial hepatectomy. Several studies including the recent research prove that liver regeneration relates to specific profiles of miRNA expression changes which are correlated to expression changes in target genes that play an important role in liver regeneration process. The processes of controlling cell number, hemostasis, and metabolism can offer new approaches for modifying miRNA expression to optimize existing tissue engineering tools. Several disease-specific miRNA expression profiles have already been identified in the liver. For example, studies have identified alcoholic liver disease, nonalcoholic steatohepatitis-associated miRNAs, and differential miRNA expression profiles in patients with primary biliary.

\subsection{Hepatocytes}

Song et al. (2010) were the first researchers who identified a miRNA-dependent mechanism for hepatocyte proliferation. They performed a genomewide analysis of miRNA expression changes through liver regeneration following a $2 / 3$ partial hepatectomy in mice, which showed induction of miR-21 and repression of miR-378 through the liver regeneration peak. Based on miRNA knockdown, supplementation revisions, and luciferase tests with Hepa1,6 mouse hepatoma cells, the researchers demonstrated that miR-21 directly inhibits Btg2, a cell cycle inhibitor that prevents activation of forkhead boxM1, and indicated that miR-378 openly inhibits ornithine decarboxylase, both known to market DNA synthesis in hepatocytes after 2/3 partial hepatectomy. Both miRNAs could then be suitable candidates for modification of hepatocyte proliferation in liver tissue-engineering applications 
(Song et al., 2010). Ng et al. (2012) from exactly the same group further focused on the role of miR-21 in mouse liver regeneration. They proposed a miR-21 dependent mechanism for translation of cyclin D1 in early phase of liver regeneration. MiR-21 inhibits RHOB expression and thereby facilitates Akt1mediated activation of mTORC1 that promotes cyclin D1 translation initiation. They postulated that the event ofmiR-21 in mouse liver regeneration is to market cyclin D1 translation, ultimately causing progression through G1 and to the S phase of the cell cycle. Although their researches primarily address the mechanisms behind themiR-21modulated cyclin D1 translation during liver regeneration, these results further boost the attractiveness of utilizing miR-21 as a possible tool in hepatic tissue engineering, e.g. by transitional up regulation for induction of hepatocyte proliferation $(\mathrm{Ng}$ et al., 2012). In a recently published study by Yan et al. (2014) further evidence was as long as miR-21 accelerates hepatocyte proliferation. The group performed researches with primary mouse hepatocytes and indicated thatmiR-21 was up regulated under stimulation with epidermal growth factor, while hepatocytes quickly entered the Sphase of the cell cycle (after transfection with miR21: $20 \%$ of cells in S-phase; control: $10 \%$ of cells in S-phase). Chances are they investigated the partnership between miR-21 and PI3K/Akt signaling. Their data indicate thatmiR-21 overexpression in primary hepatocytes increased Akt expression levels and activation (p-Akt); equally, low miR-21 expression decreased Akt expression and activation, and PTEN mRNA and protein levels were up- or down regulated, separately, suggesting that miR-21 promoted PI3K/Akt activation in a PTEN-dependent manner in primary hepatocytes in vitro. These email facts are on the other hand to the findings of $\mathrm{Ng}$ et al. (2012) who suggested that PTEN accumulation didn't inhibit Akt1 activation in liver regeneration. Cirera-Salinas et al. (2012) investigated the miR-33 family, that will be which can regulate cholesterol and fatty acid metabolism in concert making use of their host genes. They indicated that overexpression of miR-33 induced a substantial G1 cycle arrest in the human hepatoma cell line HuH7 and the human alveolar epithelial cell line A439 by decreasing CCND1 and CDK6 mRNA levels. Both genes had previously been identified as targets of miR-33. Consistently, the inhibition of miR-33 in mice using phosphorothioate backbone antisense oligonucleotides improved liver regeneration after partial hepatectomy. After a month of treatment with miR-33 inhibitors, the hepatic expression of miR-33 was decreased by more than $50 \%$. In these animals, the human body weight recovery was 1.5 fold increased at $96 \mathrm{~h}$ after partial hepatectomy compared to controls treated with control anti-miRNA, indicating that liver regeneration was markedly increased by miR-33 inhibition. Zhou et al. (2012) investigated the role of miR-26a for hepatocyte proliferation. MiR-26a has been identified to be down regulated during liver regeneration in many rodent studies, both in mice and in rats. Utilizing a similar approach as CireraSalinas et al. (2012), they treated mice with miR-26a mimics and oligonucleotides and showed that artificial down regulation of miR-26a enhanced hepatocyte proliferation (1.2 fold increase of Ki-67 positive cells in comparison to controls) and induced up regulation of the potential targets cyclin D2 and cyclin E2. Chen et al. (2011) have proven that overexpression of miR-26a down-regulated cyclin E2 protein levels and significantly can decrease proliferation of HepG2 cells. Similar results regarding induction of hepatocyte proliferation were obtained by Pan et al. (2012) for miR-127 which was observed to be down regulated 3 and $24 \mathrm{~h}$ after partial hepatectomy in rats. Using immortalized BRL-3A rat liver cells, they supply evidence that down regulation of miR-127 may be as a result of rapid methylation of its promoter during the very first $24 \mathrm{~h}$ after partial hepatectomy. They assumed that repression of miR-127 might subscribe to the regulation of liver cell proliferation via Bcl6mediated antagonism to the p53-mediated induction of CDKN1A during the very first $24 \mathrm{~h}$ of liver regeneration after partial hepatectomy. Yuan et al. (2013) focused on miR-211, which will be frequently up regulated in hepatocellular carcinoma since the replication of hepatocytes in liver regeneration and uncontrolled proliferation of tumor cells in hepatocellular carcinoma in many cases are modulated by common regulatory pathways. They first demonstrated thatmiR-211 enhanced proliferation in cultured mouse hepatocytes. MiR211 and control mimic miRNAs had therefore been transfected to the cultured cells that have been supplemented with epidermal growth factor to induce hepatocyte proliferation. From the mechanistic perspective, they demonstrated that miR- 211 overexpression led to rapid S-phase entry of hepatocytes during liver regeneration. At $36 \mathrm{~h}$ after partial hepatectomy, the percentage of proliferating cells was almost doubled in miR-211 transfected animals in comparison to controls. Aryl hydrocarbon nuclear translocator mRNA was identified as a story target of miR-221, which plays a part in the proliferative activity of miR-221. In comparison to one other miRNAs, miR-211 overexpression had the strongest outcome on hepatocyte proliferation. This result could be as a result of proven fact that miR-211 overexpression was induced with lentiviral particles, which however cannot immediately be translated to the clinic. The differentiation of stem cells or other forms of progenitor cells to hepatocyte-like cells is the 2nd major field where miRNAs are actually used with potential applications for liver tissue engineering. Doddapaneni et al. (2013) were the first to make use of this approach. By emphasizing the hepatocytespecific miR-122, they first observed a gradual up regulation of miR-122 expression from human fetal liver tissues at different gestational weeks. They then transfected miR-122 mimics and controls into human fetal liver stem/progenitor cells, which 
resulted in enhanced expression of the hepatocytespecific genes alpha fetoprotein and albumin (20 and 31 fold higher in comparison to controls 21 days after transfection, respectively) and the stagespecific hepatocyte differentiation genes HNF4a and $\mathrm{C} / \mathrm{EBP} \alpha$ (6.9 fold and 5.5fold higher in comparison to controls) in the miR-122-supplemented cells. The writers concluded that overexpression of miR-122 in stem/progenitor cells caused up regulation of the expression of genes coding for hepatocyte-specific functions during differentiation of fetal liver stem/progenitor cells towards hepatic lineage specification. To be able to test for a more practical approach that may potentially be translated to clinical use, Davoodian et al. (2014a) performed similar experiments in human adipose tissuederived stem cells (hA-DSCs) because human fetal liver stem/progenitor cells really are a very limited cell source. They demonstrated that hepatic differentiation could be improved by the overexpression of miR-122 (albumin and HNF4a mRNA levels: 3fold higher in miR-122 transfected cells in comparison to controls). However, hepatic differentiation was less effective in hA-DSCs when compared to human fetal liver cells. Deng et al. (2014) further mechanistically investigated the role of miR-122 in hepatic differentiation. They first investigated miR-122 levels during maturation of mouse embryonic stem cells towards hepatocytes and found a gradual increase through the induction stages. Then, they indicated that overexpression of miR-122 promoted hepatic differentiation and maturation of murine embryonic stem cells. FoxA1 and HNF4amRNAwere significantly up regulated aftermiR-122 transfection (3fold and 2fold higher expression in comparison to controls after 9 days of hepatic induction with activin A, FGF-4, and sodium butyrate). They concluded that overexpression of miR-122 at a proper stage could promote hepatic differentiation and maturation by regulating the total amount between proliferation and differentiation as well as the total amount between epithelial-to-mesenchymal transition and mesenchymal-to-epithelial transition, partially through amiR-122/FoxA1/HNF4a-positive feedback loop. This observation was supported by the findings of Tanimizu et al. (2014) screening that miR-122 restricts the hepatic differentiation potential of adult liver stem/progenitor cells (Tanimizu et al., 2014). Alizadeh et al. (2015) studied the role of let-7b in the regulation of hepatic specific factors in human adipose-tissue-derived mesenchymal stem cells (hAT-MSCs). This miRNA was chosen on the basis of the expression profiles of mRNAs and microRNAs in human MSCs. They indicated that the expression of the liver-enriched transcription factors HNF4a and HNF6 was conversely correlated with those of let-7 miRNAs during hepatic differentiation of hAT-MSCs. Inhibition of let-7b caused up regulation of liverenriched transcription factors in hAT-MSCs, a rise in the expression of miR-122 emulating the options that come with functional hepatocytes, and accumulation of these cells in the G0/G1 phase of the cell cycle $(90 \%$ in let-7b-inhibited cells vs. $72 \%$ in control group), that was proposed to trigger the initiation of hepatic commitment. Davoodian et al. (2014b) further investigated the role of miRNAs of the let-7 family for differentiation of hA-DSCs. They indicated that HNF4a, ALB, AFP, CK18, and CK19 expression was significantly up regulated in hA-DSCs transduced with let-7f inhibitor coding lentiviruses followed by increases in urea production and glycogen deposition. They concluded that inhibition of let-7f could facilitate induction of hA-DSCs into hepatocyte-like cells. Since they performed viral transduction for miRNA manipulation, let-7f depletion was persistent through the study period of 21 days. The results on HNF4a and albumin mRNA levels were comparable to the outcomes obtained by miR-122 supplementation with the exact same kind of cells (Davoodian et al., 2014b). Möbus et al. (2015) turned from human adipose tissue-derived stem cells to mouse embryonic stem cells, as they'd previously shown that embryonic stem cells or induced pluripotent stem cells may be used to generate partially functional hepatocytes. To be able to improve hepatic differentiation protocols by making use of miRNAs, they first compared the miRNA expression profiles of mouse hepatocyte-like cells and mature and fetal livers. In a next step, out of 20 differentially expressed miRNAs, only miR-199a$5 p$ was identified to have the ability to enhance the in vitro hepatic differentiation from murine as well as human embryonic stem cells. They revealed that inhibition of miR- 199a-5p in human ESC-derived HLCs enhanced the engraftment and repopulation capacity in the liver of immunodeficient fumarylacetoacetate knockout mice and identified SMARCA4 and MST1 as novel targets of miR-199a-5p that may subscribe to improved hepatocyte generation and in vivo liver repopulation. Significantly higher quantities of human albumin were detectable in mice transplanted with miR199a-5p inhibited mouse ESCs set alongside the control group. However, the writers noted that liver repopulation with miR-199a-5p-modified hepatocyte-like cells remained less efficient than primary human hepatocytes, helping to make further improvements of protocols for generation of hepatocyte-like cells from embryonic cells or the investigation of other sources mandatory. Another approach to optimize hepatic differentiation is to utilize a group of miRNAs in place of an individual miRNA. In an original screening study on miRNAs during hepatic differentiation of human umbilical cord lining derived MSCs (hMSCs), Cui et al. (2012; 2013) observed that miR-1246, miR-1290, miR148a,miR-30a, miR-424 andmiR-542-5pwere not just consistently overexpressed but specifically associated with hepatic (vs. Osteogenic) differentiation. To be able to investigate whether these miRNAs can be used for hepatic differentiation, then they performed overexpression studies with each of these six miRNAs. While an individual miRNA (or liver-enrichedmiR-122) couldn't initiate hepatic differentiation, ectopic overexpression of seven 
miRNAs (miR-1246, miR-1290, miR-148a, miR-30a, miR-424, miR-542-5p, and miR-122) induced hMSC conversion into functionally mature hepatocytes. In proof of concept studies with a CCL4-induced liver injury model, they demonstrated that transplantation of the so called "iHep cells" improved liver function and could restore the injured livers. Set alongside the other approaches, the effect of the miRNA cocktail on the gene expression levels is apparently at the least similarly effective. However, a primary comparison of different approaches, i.e. supplementation or depletion of a certain miRNA vs. a set of miRNAs would of course only be possible when both approaches are directly compared in the same study under identical conditions (Table 1).

Table 1: The liver's microRNAs

\begin{tabular}{|c|c|}
\hline miRNA & Aim \\
\hline Let7-b & \\
Let7-f & Differentiation \\
miR-122 & \\
miR-199a-5p & \\
\hline miR-21 & \\
miR-26a & Proliferation \\
miR-33 & \\
miR-127 & \\
\hline
\end{tabular}

\section{Method}

In this study, researchers converted hMSCs into hepatocytes in a direct manner using a seven-miRNA combination. Compared to conventional methods, the seven-miRNA combination promoted hepatic differentiation of hMSCs in an efficient manner. For example, after 6 days of induction, growth factors up regulated albumin expression approximately 4-fold, whereas the seven-miRNA combination up regulated albumin expression 10-fold. Furthermore, the uptake of Ac-Dil-LDL and the storage of glycogen by hMSCs were induced by the seven-miRNA combination after 6 days, whereas these hepatic functions were not observed in the conventional induction group until 14 days and after induction. Furthermore, the seven-miRNA combination can alter the cell morphology from fibroblast-like to epithelial. This phenomenon wasn't observed in the conventional induction group. Therefore, compared to the conventional induction method, seven-miRNA combination is an easier, faster, and more efficient way of hepatic enzyme induction.

\section{Conclusion}

MicroRNAs (miRNA) comprise a small group of non-coding small RNAs (17-25 nt) which are consistent with post-transcriptional regulation process and can be identified in different plants and animals (Subramanian et al., 2010). They have an important role in the liver. Moreover, miRNAs control hepatocyte proliferation over liver regeneration and have an important role in modulating proliferation and cell cycle progression genes after hepatectomy. The results of the recent research indicated that miR-1246, miR-1290, miR148a, miR-30a, miR-424 and miR-542-5p were definitely overexpressed during hepatic differentiation of hMSCs. In other words, MiR-1246 plays an important role in DNA damage, regulates chloride transport, and can be used as a circulating biomarker of epithelial cells. MiR-1290 impairs cytokinesis and affects the reprogramming of cancerous colon cells. MiR-30a inhibits the epithelial-mesenchymal transition (EMT) by targeting Snai1, and the miR-30 family is necessary for vertebrate hepatobiliary development. MiR-148a is a promising candidate for an earlier, stable and sensitive biomarker of rejection and hepatic damage after liver transplantation. MiR-424 controls human monocyte/macrophage differentiation (Wang et al., 2009). The main focus of this research is on miR542-5p and its important role in tumorigenesis and in the cellular senescence program of human diploid fibroblasts. According to the results, these six miRNAs have key roles in the HGF-induced hepatic differentiation of hMSCs. MiR-122 is highly expressed in the liver where it constitutes $70 \%$ of the sum total miRNA pool. MiR-122 participates in cholesterol metabolism and hepatocellular carcinoma formation. Overexpression of miR-122 enhances in vitro hepatic differentiation of fetal liver-derived stem/progenitor cells but whether miR-122 can initiate hepatic differentiation of hMSCs remains unknown. Here the researchers proved that hMSCs can be converted into functional hepatocytes only if all the miRs (miR-122, miR-1246, miR-1290, miR-148a, miR-30a, miR-424, and miR-542-5p) are used.

\section{References}

Alizadeh E, Akbarzadeh A, Eslaminejad MB, Barzegar A, Hashemzadeh S, Nejati-Koshki K, and Zarghami N (2015). Up Regulation of Liver-enriched Transcription Factors HNF4a and HNF6 and Liver-Specific MicroRNA (miR-122) by Inhibition of Let-7b in Mesenchymal Stem Cells. Chemical Biology \& Drug Design, 85(3): 268-279.

Callegari E, Gramantieri L, Domenicali M, D'Abundo L, Sabbioni S and Negrini M (2015). MicroRNAs in liver cancer: a model for investigating pathogenesis and novel therapeutic approaches. Cell Death and Differentiation, 22(1): 46-57.

Chen X, Murad M, Cui YY, Yao L J, Venugopal SK, Dawson $\mathrm{K}$, and $\mathrm{Wu} J$ (2011). miRNA regulation of liver growth after 50\% partial hepatectomy and small size grafts in rats. Transplantation, 91(3): 293-299.

Cirera-Salinas D, Pauta M, Allen RM, Salerno AG, Ramírez C M, Chamorro-Jorganes A, and Baldan A (2012). Mir-33 regulates cell proliferation and cell cycle progression. Cell Cycle, 11(5): 922-933. 
Costa FF (2010). Epigenomics in cancer management. Cancer Management and Research, 2: 255-265.

Cui L, Shi Y, Zhou X, Wang X, Wang J, Lan Y, and Zhang J (2013). A set of microRNAs mediate direct conversion of human umbilical cord liningderived mesenchymal stem cells into hepatocytes. Cell Death and Disease, 4(11): e918.

Cui L, Zhou X, Li J, Wang L, Wang J, Li Q, and Shi Y (2012). Dynamic microRNA profiles of hepatic differentiated human umbilical cord liningderived mesenchymal stem cells. PLoS One, 7(9): e44737.

Davoodian N, Lotfi AS, Soleimani M, and Mowla SJ (2014a). MicroRNA-122 Overexpression Promotes Hepatic Differentiation of Human Adipose Tissue-Derived Stem Cells. Journal of Cellular Biochemistry, 115(9): 1582-1593.

Davoodian N, Lotfi AS, Soleimani M, Mola SJ, and Arjmand S (2014b). Let-7f microRNA negatively regulates hepatic differentiation of human adipose tissue-derived stem cells. Journal of Physiology and Biochemistry, 70(3): 781-789.

Deng X G, Qiu RL, Wu YH, Li ZX, Xie P, Zhang J, and Deng JM (2014). Overexpression of miR-122 promotes the hepatic differentiation and maturation of mouse ESCs through a miR122/FoxA1/HNF4a-positive feedback loop. Liver International, 34(2), 281-295.

Doddapaneni R, Chawla Y K, Das A, Kalra JK, Ghosh S, and Chakraborti A (2013). Overexpression of microRNA-122 enhances in vitro hepatic differentiation of fetal liver-derived stem/progenitor cells. Journal of Cellular Biochemistry, 114(7): 1575-1583.

Finch ML, Marquardt JU, Yeoh GC and Callus BA (2014). Regulation of microRNAs and their role in liver development, regeneration and disease. The International Journal of Biochemistry and Cell Biology, 54: 288-303.

Kane NM, Thrasher AJ, Angelini GD and Emanueli C (2014). Concise review: MicroRNAs as modulators of stem cells and angiogenesis. Stem Cells, 32(5): 1059-1066.

Krol J, Loedige I and Filipowicz W (2010). The widespread regulation of microRNA biogenesis, function and decay. Nature Reviews Genetics, 11(9): 597-610.

Möbus S, Yang D, Yuan Q, Lüdtke THW, Balakrishnan A, Sgodda M, and Manns MP (2015). MicroRNA199a-5p inhibition enhances the liver repopulation ability of human embryonic stem cell-derived hepatic cells. Journal of Hepatology, 62(1): 101-110.
Ng R, Song G, Roll GR, Frandsen NM, and Willenbring $H$ (2012). A microRNA-21 surge facilitates rapid cyclin D1 translation and cell cycle progression in mouse liver regeneration. The Journal of Clinical Investigation, 122(3): 1097-1108.

Pan C, Chen H, Wang L, Yang S, Fu H, Zheng Y, and Jiao B (2012). Down-regulation of MiR-127 facilitates hepatocyte proliferation during rat liver regeneration. PloS One, 7(6): e39151.

Song G, Sharma AD, Roll GR, Ng R, Lee AY, Blelloch $\mathrm{RH}$, and Willenbring H (2010). MicroRNAs control hepatocyte proliferation during liver regeneration. Hepatology, 51(5): 1735-1743.

Sontheimer EJ and Carthew RW (2005). Silence from within: endogenous siRNAs and miRNAs. Cell, 122(1): 9-12.

Subramanian S and Steer CJ (2010). MicroRNAs as gatekeepers of apoptosis. Journal of Cellular Physiology, 223(2): 289-298.

Tanimizu N, Kobayashi S, Ichinohe N, and Mitaka T (2014). Downregulation of miR122 by grainyhead-like 2 restricts the hepatocytic differentiation potential of adult liver progenitor cells. Development, 141(23): 4448-4456.

Tzur G, Israel A, Levy A, Benjamin H, Meiri E, Shufaro $Y$, and Bentwich Z (2009). Comprehensive gene and microRNA expression profiling reveals a role for microRNAs in human liver development. PloS one, 4(10): e7511.

Wang Y and Blelloch R (2009). Cell cycle regulation by MicroRNAs in embryonic stem cells. Cancer Research, 69(10): 4093-4096.

Xi S, Yang M, Tao Y, Xu H, Shan J, Inchauste S, and Schrump DS (2010). Cigarette smoke induces C/EBP- $\beta$-mediated activation of miR-31 in normal human respiratory epithelia and lung cancer cells. PLoS One, 5(10): e13764.

Yan-nan B, Zhao-yan Y, Li-xi L, Qing-jie X, and Yong Z (2014). MicroRNA-21 accelerates hepatocyte proliferation in vitro via PI3K/Akt signaling by targeting PTEN. Biochemical and Biophysical Research Communications, 443(3): 802-807.

Yuan Q, Loya K, Rani B, Möbus S, Balakrishnan A, Lamle J, and Cantz T (2013). MicroRNA-221 overexpression accelerates hepatocyte proliferation during liver regeneration. Hepatology, 57(1): 299-310.

Zhou J, Ju W, Wang D, Wu L, Zhu X, Guo Z, and He X (2012). Down-regulation of microRNA-26a promotes mouse hepatocyte proliferation during liver regeneration. PloS one, 7(4): e33577. 